\title{
Acoustic Characterization of Polydimethylsiloxane for Microscale Acoustofluidics
}

\author{
Xu, Guangyao; Ni, Zhengyang; Chen, Xizhou; Tu, Juan; Guo, Xiasheng; Bruus, Henrik; Zhang, Dong
}

Published in:

Physical Review Applied

Link to article, DOI:

10.1103/PhysRevApplied.13.054069

Publication date:

2020

Document Version

Publisher's PDF, also known as Version of record

Link back to DTU Orbit

Citation (APA):

Xu, G., Ni, Z., Chen, X., Tu, J., Guo, X., Bruus, H., \& Zhang, D. (2020). Acoustic Characterization of Polydimethylsiloxane for Microscale Acoustofluidics. Physical Review Applied, 13(5), [054069]. https://doi.org/10.1103/PhysRevApplied.13.054069

\section{General rights}

Copyright and moral rights for the publications made accessible in the public portal are retained by the authors and/or other copyright owners and it is a condition of accessing publications that users recognise and abide by the legal requirements associated with these rights.

- Users may download and print one copy of any publication from the public portal for the purpose of private study or research.

- You may not further distribute the material or use it for any profit-making activity or commercial gain

- You may freely distribute the URL identifying the publication in the public portal 


\title{
Acoustic Characterization of Polydimethylsiloxane for Microscale Acoustofluidics
}

\author{
Guangyao Xu๑, ${ }^{1}$ Zhengyang Ni, ${ }^{1}$ Xizhou Chen, ${ }^{1}$ Juan $\mathrm{Tu},{ }^{1}$ Xiasheng Guo $\odot,{ }^{1, \dagger}$ Henrik Bruus $\odot,{ }^{2}$ and \\ Dong Zhang $\oplus^{1,3, *}$ \\ ${ }^{1}$ Key Laboratory of Modern Acoustics (MOE), Department of Physics, Collaborative Innovation Center of \\ Advanced Microstructure, Nanjing University, Nanjing 210093, China \\ ${ }^{2}$ Department of Physics, Technical University of Denmark, DTU Physics Building 309, \\ Kongens Lyngby, DK-2800, Denmark \\ ${ }^{3}$ The State Key Laboratory of Acoustics, Chinese Academy of Science, Beijing 10080, China
}

(Received 2 December 2019; revised manuscript received 24 February 2020; accepted 28 April 2020; published 27 May 2020)

\begin{abstract}
Polydimethylsiloxane (PDMS) is widely used in acoustofluidic devices among other lab-on-a-chip systems, but a systematic study of its acoustic parameters is lacking, making it difficult to study the acoustic-PDMS interactions accurately during device designs and evaluations. Here the phase velocities and attenuation coefficients of longitudinal and shear acoustic waves propagating in Sylgard ${ }^{\mathrm{TM}} 184$ samples are measured throughout the frequency band from 3.0 to 7.0 MHz. Five sample groups are prepared, corresponding to different base-agent mixing ratios $\sigma$ and curing temperatures $T,(\sigma, T)=\left(10: 1,85^{\circ} \mathrm{C}\right)$, $\left(7: 1,85^{\circ} \mathrm{C}\right),\left(5: 1,85^{\circ} \mathrm{C}\right),\left(10: 1,65^{\circ} \mathrm{C}\right)$, and $\left(10: 1,100^{\circ} \mathrm{C}\right)$. The shear wave measurements involved examining the change of the reflection coefficient at a solid surface caused by the attachment of the samples. For longitudinal waves, the parameters are obtained by analyzing their round-trip transmissions inside double-layered solid-PDMS structures. Apart from these, the influence of sample aging on the concerned parameters are also examined. With the measured results, the parameters lead to fitted functions based on the Kramers-Kronig principle, which should benefit users working outside the studied frequency range. Finally, the two independent parameters in the elastic matrix of PDMS are provided.
\end{abstract}

DOI: 10.1103/PhysRevApplied.13.054069

\section{INTRODUCTION}

In the choices of biofriendly materials [1-3] for biomedical microelectromechanical systems, polydimethylsiloxane (PDMS) is chemically inert, optically transparent, thermally stable, acoustically isotropic, among other merits like low cost and easy to fabricate [4], and is therefore widely used in lab-on-a-chip systems [5]. Furthermore, PDMS also performs well in biomedical studies related to ultrasound, like photoacoustics [6], tissue fabrication [7], and acoustofluidics [8-11].

Having boomed over the past decade, acoustofluidics are characterized by high energy efficiency and good biocompatibility [12], and are therefore considered promising tools in biomedical and chemical analysis $[13,14]$ and particle manipulation $[7,15]$. The driving mechanism of acoustofluidics relies on the actuation of surface $[10,13,16$, 17] or bulk $[9,18,19]$ acoustic waves (SAWs or BAWs). In both cases, PDMS has proved to be flexible and reliable. For example, Moiseyenko and Bruus [18] demonstrated whole-system ultrasound resonance in an all-PDMS BAW device, providing an all-polymer choice for point-of-care

\footnotetext{
*dzhang@nju.edu.cn

†guoxs@nju.edu.cn
}

applications. In SAW chips, PDMS can be easily bonded to the piezoelectric substrates, such as lithium niobate $\left(\mathrm{LiNbO}_{3}\right)$ and is favored by many [13,15-17].

The design, evaluation, and optimization of such devices require dealing with wave-PDMS interactions, e.g., wave absorption, reflection, propagation, resonance, and acoustic impedance matching $[17,18,20,21]$. In the prediction of resonance frequencies in all-PDMS BAW devices, accurate parameters describing the elasticity and viscosity of PDMS are required [18]. In SAW devices, reduced descriptions of PDMS layers like the lossy-wall model cannot well approximate the behaviors of PDMS in certain cases [20, 21]. These underline the importance of a full description of the acoustic properties of PDMS, which has unfortunately been unavailable hitherto.

Acoustic propagation in PDMS involves longitudinal $(L)$ and shear $(S)$ waves. The $L$-wave velocity is typically approximately $1100 \mathrm{~m} / \mathrm{s}$, whereas the moderate damping is around $200 \mathrm{~m}^{-1}$ [22-24]. The $S$ waves show a low phase velocity of approximately $100 \mathrm{~m} / \mathrm{s}$, inducing much shorter wavelengths [25], which leads to very large computer memory requirements in numerical simulations resolving the full physics of PDMS [17]. On the other hand, the attenuation of $S$ waves in PDMS is very high, approximately $10^{5} \mathrm{~m}^{-1}$ [25]; therefore, neglect of $S$-wave 
propagations seems reasonable [26]. However, recent studies showed that $S$ waves also cause differences inside acoustofluidic channels, especially the acoustic streaming and the motion of small particles dominated by that $[17,20]$.

Regarding currently available acoustic parameters of PDMS, Tsou et al. [22] reported the velocity and attenuation coefficient of $L$ waves at 3.0, 5.0, 7.0, 9.0, and 11.0 $\mathrm{MHz}$ frequencies for samples prepared at different mixing ratios, which is the most adopted data, whereas Folds [23] reported similar data for 13 different RTV 615 PDMS samples at $0.6 \mathrm{MHz}$. The only available $S$-wave parameters were reported by Madsen et al. [25], who set the curing condition fixed. These parameters (including the PDMS density) scattered in different literature are not obtained from the same sample groups or even the same product models, making it questionable if they are coherent with each other. Furthermore, considering the different sample preparation procedures adopted by different users [5], e.g., different mixing ratios, curing temperatures, or aging periods, it is unclear whether these parameters are universally valid.

PDMS is an isotropic viscoelastic material; its acoustic parameters are supposed to be frequency dispersive [27] and can be determined from the dynamic moduli and dynamic Poisson's ratio. However, relevant tests are difficult to carry out at megahertz frequencies and have not been reported. Meanwhile, it is also convenient to describe PDMS by its $L$ - and $S$-wave velocities and attenuation coefficients, which can be achieved through acoustic measurements.

Accurate measurement of these PDMS parameters is difficult, however, especially across a wide band. For $L$ waves, attenuation measurements using the conventional contact-type pulse-echo method [28] are influenced by the transducer-sample interfaces. Immersion-type measurements $[29,30]$ raise concerns about whether the sample surfaces are strictly perpendicular to the wave paths. For $S$ waves, only contact measurements can be used, but experimental failures can originate due to high attenuation and impedance mismatch. To be specific, $S$-wave attenuation in PDMS can be as high as $100 \mathrm{~Np} / \mathrm{mm}$ [25], making transmission-based measurements next to impossible. At megahertz frequencies, a typical $S$-wave velocity in PDMS is approximately $100 \mathrm{~m} / \mathrm{s}$ [25], which combined with the density (approximately $1000 \mathrm{~kg} / \mathrm{m}^{3}$ ) [21] leads to an acoustic impedance of approximately 0.1 MRayl. In contrast, the ceramic matching layers of commercial $S$-wave transducers have shear acoustic impedances of approximately 35 MRayl (from Olympus NDT), indicating that $S$ waves can hardly penetrate the transducer-PDMS interface.

In this work, two methods are setup to obtain the phase velocities and attenuation coefficients of $L$ and $S$ waves in PDMS across the 3.0-7.0 MHz frequency band. In the first method, the $S$-wave parameters are obtained by comparing the wave reflections at a solid surface before and after PDMS samples are attached. The $L$-wave parameters are obtained using the second approach, by interrogating wave transmissions in double-layered solid-PDMS structures. Experiments using different mixing ratios and curing temperatures are carried out, and the influence of material aging is examined.

\section{METHODS}

\section{A. The experimental scheme}

The PDMS samples are made of Sylgard 184 (from Dow Corning, USA), which is a two-part silicone elastomer. Before the experiments, five sample groups are prepared using different base-agent volume mixing ratios $\sigma$ and different curing temperatures $T,(\sigma, T)=\left(10: 1,85^{\circ} \mathrm{C}\right)$, $\left(7: 1,85^{\circ} \mathrm{C}\right),\left(5: 1,85^{\circ} \mathrm{C}\right),\left(10: 1,65^{\circ} \mathrm{C}\right)$, and $\left(10: 1,100^{\circ} \mathrm{C}\right)$. Each group consists of three disclike samples of diameter $d_{P}=50 \mathrm{~mm}$ and thickness $D_{P}=2 \mathrm{~mm}$ (the accurate thickness of each sample is calibrated using a vernier caliper), whereas every sample is used for nine replicate measurements. After the fabrication processes, all samples are kept at a room temperature of $25^{\circ} \mathrm{C}$ for 10 days before use (except those used in Sec. III F).

The two different methods share the same experimental setup illustrated in Fig. 1(a). Pulse signals (32 $\mu \mathrm{J}$, $1 \mathrm{kHz}-200 \mathrm{MHz}$ ) generated by a pulser-receiver (5900PR, Olympus NDT) are used to excite an A107S (or V155) transducer (5.0 MHz central frequency, Olympus NDT) to excite $L$ (or $S$ ) waves. The transducer is in contact with a fused silica (FS) disc (with diameter $d_{F}=59 \mathrm{~mm}$,
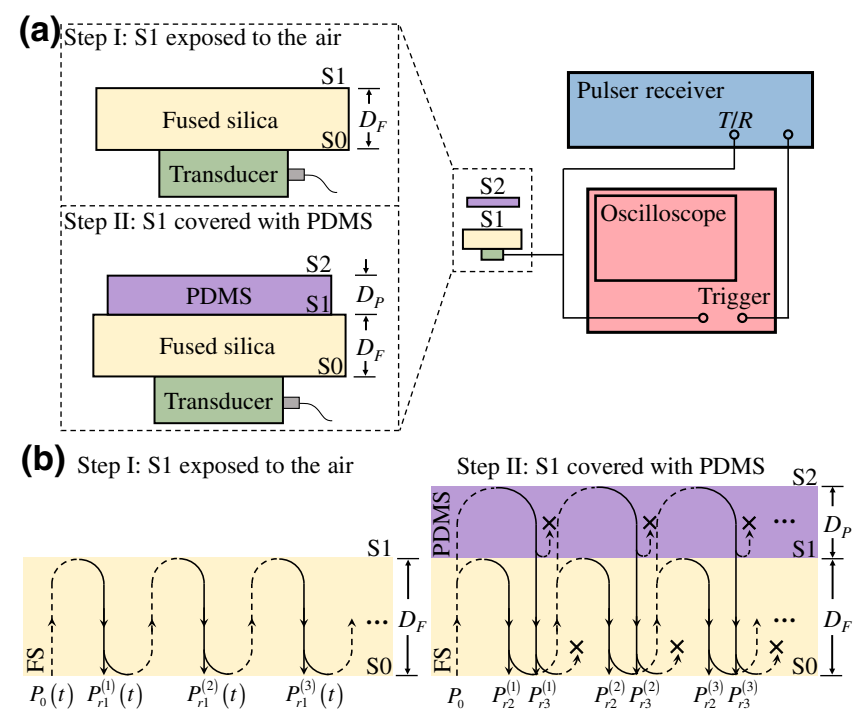

FIG. 1. (a) The experimental setup shared by both measurement methods (A and B). (b) Illustrations of the wave paths in the FS disk (yellow) and the PDMS sample (purple). $T / R$, transmit or receive. 
and thicknesses $D_{F}=7.90 \mathrm{~mm}$ for $S$-wave measurements and $D_{F}=19.64 \mathrm{~mm}$ for $L$-wave measurements), coupled through the SWC-2 couplant (Olympus NDT). Waves reflected back to the transducer are recorded before and after each sample is attached to S1 (the upper surface of the FS disc). The FS disk helps to ensure proper resolution of different reflected wave packages, and to create a difference measurement between the unloaded and PDMSloaded upper FS surfaces. The recorded signals, averaged over 256 sequences, are acquired through a digital oscilloscope (DSO9064A, Keysight, USA) at a sampling rate of 200.0 MHz. All experiments are conducted under a room temperature of $25^{\circ} \mathrm{C}$.

The detailed wave transmission paths are illustrated in Fig. 1(b), in which only the pulses drawn in solid lines can be detected by the transducers. In step I, an initial pulse $P_{0}(t)$ enters the FS disc through the interface S0, where $t$ is the time. After it impinges on $\mathrm{S} 1$, a reflected pulse $P_{r 1}(t)$ propagates back and is collected at the transducer. Then, part of the signal is reflected at S0 and again travels toward $\mathrm{S} 1$; this cyclic process continues, accompanied by a gradual decrease in signal amplitudes. Here, each forth and back signal group is assigned an index $n$, i.e., $P_{0}^{(n)}(t)$ and $P_{r 1}^{(n)}(t)$ denote the $n$th acoustic pulses going upward and downward, respectively, with $n=1,2,3, \ldots$.

In step II, a PDMS sample attached to S1 lowers the amplitude of the $P_{r 1}^{(n)}(t)$ pulses, which are renamed $P_{r 2}^{(n)}(t)$. For each $P_{0}^{(n)}(t)$ traveling upward from the bottom S0, the pulse can also complete a round-trip between S0 and S2 (across the FS and PDMS discs), which we record as $P_{r 3}^{(n)}(t)$ pulses at the transducer. All other pulses following more complicated paths are marked with crosses in Fig. 1(b), and are discarded in signal processing. It should be mentioned that the thicknesses of the FS and PDMS discs are carefully chosen so that most of the marked "harmful" pulses are separated from the desired pulses in the collected signals.

The density of PDMS is obtained by measuring the sample volumes $\left(V_{P}\right)$ and masses $\left(m_{P}\right)$ as $\rho_{P}=m_{P} / V_{P}$. In determining $V_{P}$, each sample is suspended in water, whose container is on an electrical balance. According to Archimedes's principle, the detected mass difference $(\Delta m)$ caused by sample immersion gives the sample volume as $V_{P}=\Delta m / \rho_{w}$, with $\rho_{w}=997 \mathrm{~kg} / \mathrm{m}^{3}$ being the density of water [31]. Upon removal from the container, the samples are cleaned with dust-free tissues to remove the adherent water and then placed on the balance for mass measurements. This process is repeated three times for each sample.

\section{B. The reflection method $(\operatorname{method} \mathrm{A})$}

This method involves measuring the reflection coefficients at S1 before and after the PDMS samples are attached, and is in principle valid for both $L$ - and $S$-wave measurements. The general principle is similar to that proposed by Frizzell et al. [32], but the theory and experiments are extended to broadband here, and a signal processing scheme is developed for error reductions.

Before and after a PDMS sample is attached to the FS, we measure the reflection coefficient from the FS side of $\mathrm{S} 1$, denoted by $R_{\mathrm{I}}$ and $R_{\mathrm{II}}$, respectively, which are functions of the frequency $f$. In each measurement, the signal received by the transducer is the sum of multiple arriving paths $P=\sum P_{r 1}^{(n)}(t)$, and an example of a real measurement of $P$ is given in Fig. 2. The individual paths can be seen in Fig. 2 as segmented pulses, each labeled $P_{r 1}^{(n)}(t)$. Each $P_{i}(t), i=0, r 1, r 2$, can be defined in the Fourier domain with amplitude $A_{i}(f)$ and phase $\phi_{i}(f)$. Let $\Psi_{u}(f)$ and $\Psi_{d}(f)$ be the transfer spectrum from the transducer to $\mathrm{S} 1$ and $\mathrm{S} 1$ to the transducer. Then the frequency dependent $R_{\mathrm{I}}$ and $R_{\mathrm{II}}$ are determined as

$$
\begin{gathered}
R_{\mathrm{I}}(f) \equiv \frac{\mathscr{F}\left[P_{r 1}(t)\right] / \Psi_{d}(f)}{\mathscr{F}\left[P_{0}(t)\right] / \Psi_{u}(f)}=\frac{A_{r 1}(f) e^{j \phi_{r 1}(f)} / \Psi_{d}(f)}{A_{0}(f) e^{j \phi_{0}(f)} / \Psi_{u}(f)}, \\
R_{\mathrm{II}}(f) \equiv \frac{\mathscr{F}\left[P_{r 2}(t)\right] / \Psi_{d}(f)}{\mathscr{F}\left[P_{0}(t)\right] / \Psi_{u}(f)}=\frac{A_{r 2}(f) e^{j \phi_{r 2}(f)} / \Psi_{d}(f)}{A_{0}(f) e^{j \phi_{0}(f)} / \Psi_{u}(f)},
\end{gathered}
$$

where $j=\sqrt{-1}$ and $\mathscr{F}$ is the Fourier operator. Since $R_{\mathrm{I}}$ and $R_{\mathrm{II}}$ should be independent of the index $n$, we omit the superscript $(n)$ in each pulse component.

Considering the continuity conditions across $\mathrm{S} 1$, the wave velocity $c_{P}(P=L$ or $S$ for longitudinal or shear waves) and attenuation coefficient $\alpha_{P}$ are [25]

$$
c_{P}(f)=\frac{4 \pi f A}{-B+\sqrt{B^{2}-4 A C}},
$$
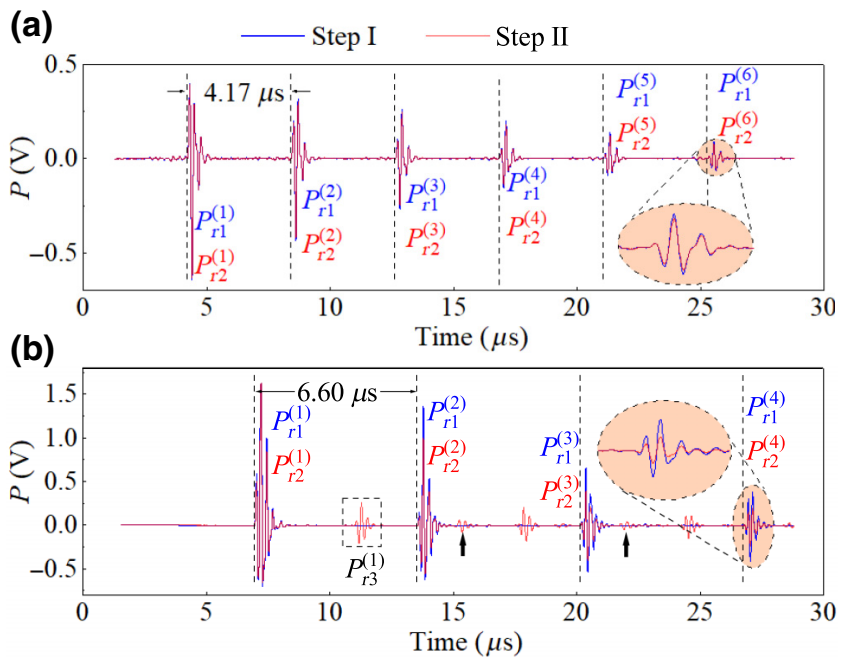

FIG. 2. Typical signals recorded by the transducers in (a) $S$ wave measurements and (b) $L$-wave measurements. 


$$
\alpha_{P}(f)=2 \pi f \sqrt{-\frac{1}{c_{P}^{2}}+\frac{2 \rho_{P}}{\rho_{F} c_{F} c_{P}}\left(\frac{1+R_{\mathrm{II}}^{*} R_{\mathrm{II}}}{1-R_{\mathrm{II}}^{*} R_{\mathrm{II}}}\right)-\frac{\rho_{P}^{2}}{\rho_{F}^{2} c_{F}^{2}}},
$$

where an asterisk denotes complex conjugate, $c_{F}$ and $\rho_{F}$ are the wave velocity and material density in FS, and

$$
\begin{gathered}
A=1+\left(\frac{1+R_{\mathrm{II}}^{*} R_{\mathrm{II}}}{1-R_{\mathrm{II}}^{*} R_{\mathrm{II}}}\right)^{2} \tan ^{2} \phi_{r}, \\
B=-\frac{4 \pi \rho_{P} f}{\rho_{F} c_{F}}\left(\frac{1+R_{\mathrm{II}}^{*} R_{\mathrm{II}}}{1-R_{\mathrm{II}}^{*} R_{\mathrm{II}}}\right)\left(1+\tan ^{2} \phi_{r}\right), \\
C=\left(\frac{2 \pi \rho_{P} f}{\rho_{F} c_{F}}\right)^{2}\left(1+\tan ^{2} \phi_{r}\right) .
\end{gathered}
$$

In step I, the soft boundary S1 leads to $R_{\mathrm{I}}(f)=-1$ and

$$
R_{\mathrm{II}}(f) \equiv \frac{\mathscr{F}\left[P_{r 2}(t)\right]}{\mathscr{F}\left[P_{r 1}(t)\right]}=-\frac{A_{r 2}(f) e^{j \phi_{r 2}(f)}}{A_{r 1}(f) e^{j \phi_{r 1}(f)}},
$$

and the amplitude and phase spectra of $R_{\mathrm{II}}(f)$ are given by

$$
\left|R_{\mathrm{II}}(f)\right|=\frac{A_{r 2}(f)}{A_{r 1}(f)}, \quad \phi_{r}(f)=\phi_{r 2}(f)-\phi_{r 1}(f)+\pi .
$$

Hence, by calculating $R_{\mathrm{II}}(f)$ through the measured $P_{r 1}(t)$ and $P_{r 2}(t), c_{P}(f)$ and $\alpha_{P}(f)$ can be determined using Eqs. (2) and (3).

\section{The round-trip transmission method (method B)}

The second method is for $L$-wave measurements, and requires only the step II configuration in Fig. 1(a). Here, the $P_{r 2}(t)$ and $P_{r 3}(t)$ pulses are considered. Omitting the superscript $(n)$, their Fourier transforms are given by

$$
\begin{gathered}
\mathscr{F}\left[P_{r 2}(t)\right]=A_{r 2}(f) e^{j \phi_{r 2}(f)} \\
=A_{0}(f) e^{j \phi_{0}(f)+2\left(j k_{F}-\alpha_{F}\right) D_{F}} R_{\mathrm{FP}}, \\
\mathscr{F}\left[P_{r 3}(t)\right]=A_{r 3}(f) e^{j \phi_{r 3}(f)} \\
=A_{0}(f) R_{\mathrm{PA}} T_{\mathrm{FP}} T_{\mathrm{PF}} \\
\times e^{j \phi_{0}(f)+2\left[\left(j k_{F}-\alpha_{F}\right) D_{F}+\left(j k_{P}-\alpha_{P}\right) D_{P}\right]},
\end{gathered}
$$

where $k_{F}$ and $k_{P}$ are the wave numbers in FS and PDMS, $\alpha_{F}$ is the attenuation coefficient in FS, $R_{\mathrm{PA}}=-1$ is the reflection coefficient at $\mathrm{S} 2, R_{\mathrm{FP}}$ and $T_{\mathrm{FP}}$ are the reflection and transmission coefficients from FS to PDMS, respectively, and $T_{\mathrm{PF}}$ is the transmission coefficient from PDMS
TABLE I. Material parameters of fused silica [33].

\begin{tabular}{lcc}
\hline \hline Parameters & Symbol & Value \\
\hline$L$-wave velocity & $c_{F, L}$ & $5950 \mathrm{~m} / \mathrm{s}$ \\
$S$-wave velocity & $c_{F, S}$ & $3760 \mathrm{~m} / \mathrm{s}$ \\
Density & $\rho_{F}$ & $2198 \mathrm{~kg} / \mathrm{m}^{3}$ \\
\hline \hline
\end{tabular}

back to FS. The reflection and transmission coefficients are given by [28]

$$
\begin{aligned}
R_{I J}= & \frac{\rho_{J} c_{J}-\rho_{I} c_{I}}{\rho_{I} c_{I}+\rho_{J} c_{J}}, \quad T_{I J}=\frac{2 \rho_{J} c_{J}}{\rho_{I} c_{I}+\rho_{J} c_{J}} \\
& \text { for } I, J=F \text { or } P .
\end{aligned}
$$

Combining Eqs. (7a) and (7b) yields

$$
\frac{A_{r 3}(f)}{A_{r 2}(f)} e^{j\left[\phi_{r 3}(f)-\phi_{r 2}(f)\right]}=\frac{T_{\mathrm{FP}} T_{\mathrm{PF}}}{-R_{\mathrm{FP}}} e^{-2\left[\alpha_{P} D_{P}+j k_{P} D_{P}\right]}
$$

The wave velocity in PDMS, $c_{P}(f)$, is then obtained from the phase difference between $P_{r 2}(t)$ and $P_{r 3}(t)$, written as

$$
c_{P}(f)=\frac{-4 \pi f D_{P}}{\phi_{r 3}(f)-\phi_{r 2}(f)} .
$$

We obtain $\alpha_{P}(f)$ from Eq. (9) as

$$
\alpha_{P}(f)=\frac{-1}{2 D_{P}} \ln \left[\frac{-R_{\mathrm{FP}}}{T_{\mathrm{FP}} T_{\mathrm{PF}}} \frac{A_{r 3}(f)}{A_{r 2}(f)}\right] .
$$

The accuracy of the determined $c_{P}(f)$ is therefore dependent on the precision in calculating $\phi_{r 3}(f)-\phi_{r 2}(f)$. The material properties of the FS listed in Table I are adopted from Ref. [33], verified through the traditional pulseecho method for wave velocities [28] and Archimedes's principle for the density.

\section{RESULTS}

\section{A. The density of PDMS}

For each of the five sample groups, the measured PDMS density is averaged and presented in Table. II, together with the standard errors. The results show that different preparation protocols do not make a significant difference to the density of PDMS, and an average value of $1030.5 \mathrm{~kg} / \mathrm{m}^{3}$ can be safely employed. This value is very close to that given by the manufacturer (1.03 times the density of water), whereas $\rho_{P}$ in the literature varies between $1070,1020,920$, and $970 \mathrm{~kg} / \mathrm{m}^{3}$ [21,23,25,31,34].

\section{B. The signal characteristics}

Typical recorded $S$ - and $L$-wave signals are shown in Figs. 2(a) and 2(b), respectively, with the blue and red lines corresponding to those detected before and after a PDMS 
TABLE II. Measured PDMS density for five sample groups.

\begin{tabular}{cccccc}
\hline \hline & $5: 1,85^{\circ} \mathrm{C}$ & $7: 1,85^{\circ} \mathrm{C}$ & $10: 1,65^{\circ} \mathrm{C}$ & $10: 1,85^{\circ} \mathrm{C}$ & $10: 1,100^{\circ} \mathrm{C}$ \\
\hline Density $\left(\mathrm{kg} / \mathrm{m}^{3}\right)$ & $1030.7 \pm 1.9$ & $1030.7 \pm 0.8$ & $1029.4 \pm 2.2$ & $1031.4 \pm 1.0$ & $1030.4 \pm 0.5$ \\
\hline \hline
\end{tabular}

sample is attached to S1. In Fig. 2(a), the $S$ waves reflect back and forth within the FS layer, whereas $P_{r 1}^{(n)}(t)$ and $P_{r 2}^{(n)}(t)$ attenuate as $n$ increases.

In Fig. 2(b), each $L$-wave pulse $P_{r 2}^{(n)}(t)$ is followed by a $P_{r 3}^{(n)}(t)$ pulse, which has a much lower amplitude for the same $n$ since the $P_{r 3}^{(n)}(t)$ pulse experiences an additional round trip inside the PDMS layer. The fact that the $P_{r 3}^{(n)}(t)$ pulses are almost absent in the $S$-wave measurements of Fig. 2(a) indicates that the attenuation of $S$ waves is far higher than that of $L$ waves in PDMS, and hence the transmission-based method B is not suitable for measuring $S$-wave parameters.

\section{The reflection coefficient $R_{\mathrm{II}}(f)$ in method $A$}

Considering the index $n$, Eq. (6) can be rewritten as

$$
\left|R_{\mathrm{II}}(f)\right|=\sqrt[n]{\frac{A_{r 2}^{(n)}(f)}{A_{r 1}^{(n)}(f)}}, \quad \phi_{r}(f)=\frac{\phi_{r 2}^{(n)}(f)-\phi_{r 1}^{(n)}(f)}{n}+\pi .
$$

To improve the accuracy of $\phi_{r}$ measurements, whose typical values were approximately $10^{-2}$, Madsen et al. [25] chose $n=10$ to calculate $R_{\mathrm{II}}(f)$. However, a large $n$ yields a low signal-to-noise ratio (SNR), which in turn impairs the precision of $\left|R_{\mathrm{II}}(f)\right|$ evaluations.

Here, a single $S$-wave measurement on a $\left(10: 1,85^{\circ} \mathrm{C}\right)$ sample is taken as an example to illustrate the data processing procedure for method A. Within the frequency range 3.0-7.0 MHz, the amplitude and unwrapped phase spectra of the $P_{r 1}^{(n)}(t)$ and $P_{r 2}^{(n)}(t)$ pulses are shown in Figs. 3(a) and $3(\mathrm{~b})$, respectively, with the former showing a peak response at about $4.2 \mathrm{MHz}$.

As can be seen from Fig. 3(a), the $A_{r 2}^{(n)}$ amplitudes are slightly smaller than the $A_{r 1}^{(n)}(f)$ amplitudes, but both decrease as $n$ increases. The $A_{r 2}^{(n)} / A_{r 1}^{(n)}$ curves are plotted as functions of $f$ in Fig. 3(c), from which the frequency dependent $\left|R_{\mathrm{II}}(f)\right|$ are determined using Eq. (12) (see the inset). As expected, the $\left|R_{\mathrm{II}}(f)\right|$ curves agree well for different $n$ values, all a value around 0.975. In Fig. 3(b), the phase spectra of $P_{r 1}^{(n)}(t)$ and $P_{r 2}^{(n)}(t)$ are quite close to each other at different $n$. In the enlarged view, the $n=4$ curves at $f=5.0 \mathrm{MHz}$ show a phase difference $\phi_{r 2}^{(4)}-\phi_{r 1}^{(4)}=$ -0.04 . To discern such small differences, $\phi_{r 2}^{(n)}-\phi_{r 1}^{(n)}$ for $n=1, \ldots, 6$ are plotted as functions of $f$ in Fig. 3(d). At $n \leq 4$, the phase difference $\phi_{r 2}^{(n)}-\phi_{r 1}^{(n)}$ shows almost the same response at different $f$, whereas it increases with increasing $n$. However, for $n=5$ and $6, \phi_{r 2}^{(n)}-\phi_{r 1}^{(n)}$ becomes highly dispersive. The phase $\phi_{r}(f)$ obtained from different $n$th pulse pairs is plotted in the inset of Fig. 3(d), which shows that larger $n$ values causes more disordered $\phi_{r}(f)$ curves, which is ascribed to the lower SNRs, as illustrated in Fig. 2(a).

Consequently, $\left|R_{\mathrm{II}}(f)\right|$ and $\phi_{r}(f)$ calculated from a single pair of $P_{r 1}^{(n)}(t)$ and $P_{r 2}^{(n)}(t)$ are highly dependent on $n$. Consulting Eq. (12), different $n$ values should lead to consistent $\left|R_{\mathrm{II}}(f)\right|$ results, inspiring the idea of choosing a combination of $n$ values, i.e., different $P_{r 1}^{(n)}(t)$ and $P_{r 2}^{(n)}(t)$ pairs are incorporated in calculations. A fitting protocol is developed by rewriting Eq. (12) as

$$
\begin{array}{r}
\log \left[A_{r 2}^{(n)}(f) / A_{r 1}^{(n)}(f)\right]=n \log \left|R_{\mathrm{II}}(f)\right|, \\
\phi_{r 2}^{(n)}(f)-\phi_{r 1}^{(n)}(f)+n \pi=n \phi_{r}(f) .
\end{array}
$$

Hence, $\left|R_{\mathrm{II}}(f)\right|$ and $\phi_{r}(f)$ can be obtained from linear fittings of $\log \left[A_{r 2}^{(n)}(f) / A_{r 1}^{(n)}(f)\right]$ and $\phi_{r 2}^{(n)}(f)-\phi_{r 1}^{(n)}(f)+n \pi$ as functions of $n$, respectively. Also, since $\phi_{r}(f)$ appears only as the argument of tangent functions in Eq. (4), the term $n \pi$ in Eq. (13) is omitted.

In order to guarantee the fitting procedure leads to optimized results, the range of $n$ values included in the fitting is carefully considered by testing all combinations
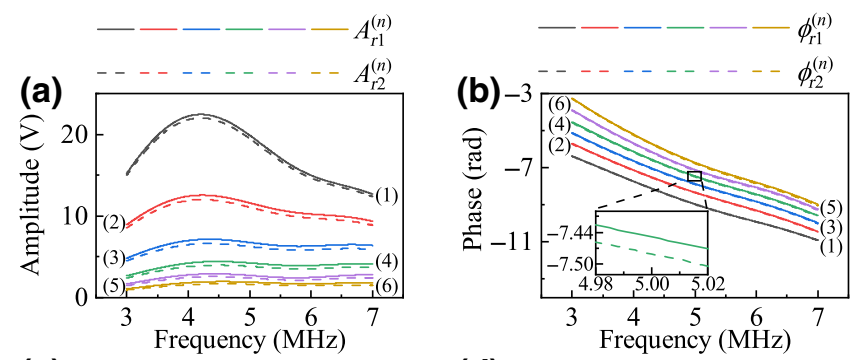

(c)
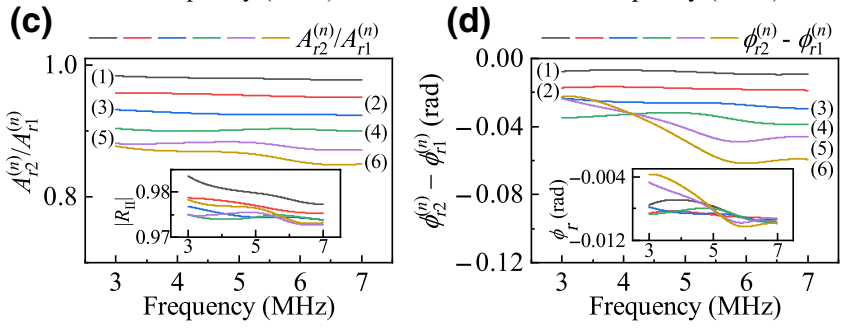

FIG. 3. (a) The amplitude and (b) the phase versus frequency of $P_{r 1}^{(n)}(t)$ and $P_{r 2}^{(n)}(t)$ pulses for $n=1,2, \ldots, 6$ from $S$-wave measurements on a $\left(10: 1,85^{\circ} \mathrm{C}\right)$ sample. (c),(d) Corresponding frequency dependencies of amplitude ratios (dimensionless) and phase differences. 

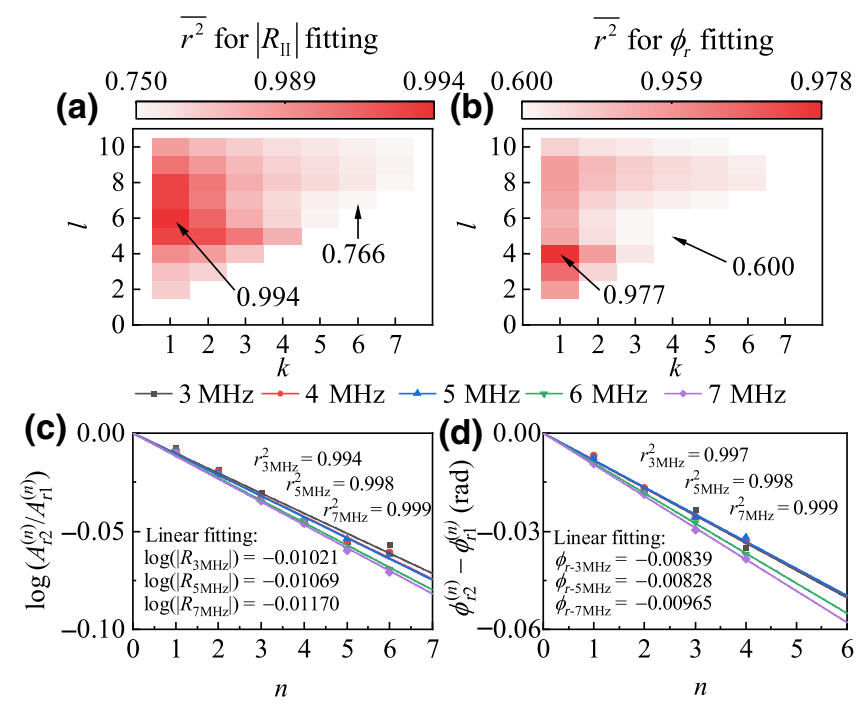

FIG. 4. The $\overline{r^{2}}$ maps in the $k-l$ space, where the $k$ th- $-l$ th pulse pairs of $P_{r 1}^{(n)}(t)$ and $P_{r 2}^{(n)}(t)$ are used for determining (a) $R_{\mathrm{II}}(f)$ and (b) $\phi_{r}^{(n)}$ through linear fitting. The fitted results of (c) $\log \left[A_{r 2}^{(n)}(f) / A_{r 1}^{(n)}(f)\right]$ and (d) $\phi_{r 2}^{(n)}(f)-\phi_{r 1}^{(n)}(f)$ as linear functions of $n$. The data are extracted from an $S$-wave measurement on a $\left(10: 1,85^{\circ} \mathrm{C}\right)$ sample.

of $k \leq n \leq l$ for $1 \leq k<l \leq 10$. For each combination, the coefficients of determination $\left(r^{2}\right)$ for the fittings are averaged across the frequency range $3.0-7.0 \mathrm{MHz}$ in steps of $2.0 \mathrm{kHz}$, obtaining $\left(\overline{r^{2}}\right)$. For the example in Fig. 3, maps of $\overline{r^{2}}$ for fitting the amplitude and phase of $R_{\mathrm{II}}(f)$ are presented in Figs. 4(a) and 4(b), respectively, and the maximum and minimum values are marked. In both figures, $\overline{r^{2}}$ decreases as $k$ and $l$ increase, indicating that pulse components with large $n$ might become unreliable in applying method $\mathrm{A}$. The best fitting combination for $\log \left[A_{r 2}^{(n)}(f) / A_{r 1}^{(n)}(f)\right]$ and $\phi_{r 2}^{(n)}(f)-\phi_{r 1}^{(n)}(f)$ are $n=$ $1,2, \ldots, 6$ and $n=1,2,3,4$, respectively. That the phases can be determined using fewer pulse pairs is explained from Fig. 3(d), where $\phi_{r}(f)$ corresponding to $n=5$ and 6 shows no systematic dependency on the frequency. Using this procedure, pulse combinations for each measurement are carefully tested, and the optimized $n$ ranges are not necessarily the same for all measurements. With the obtained $n$ combinations, linear fittings yield the amplitude and phase of $R_{\mathrm{II}}$ at five typical frequencies shown in Figs. 4(c) and 4(d), in which all the lines exhibit good linearity with $\overline{r^{2}}>0.99$. The slopes of the lines are used to obtain $\left|R_{\mathrm{II}}(f)\right|$ and $\phi_{r}(f)$ based on Eq. (13), whereas their decrease with increasing $f$ is a clear indication of the frequency dispersive nature of $R_{\mathrm{II}}(f)$.

Based on the same fitting scheme, data processing for an $L$-wave measurement is also given here for comparison. The amplitude and unwrapped phase spectra of the $P_{r 1}^{(n)}(t)$ and $P_{r 2}^{(n)}(t)$ pairs are given in Figs. 5(a) and 5(b), with the
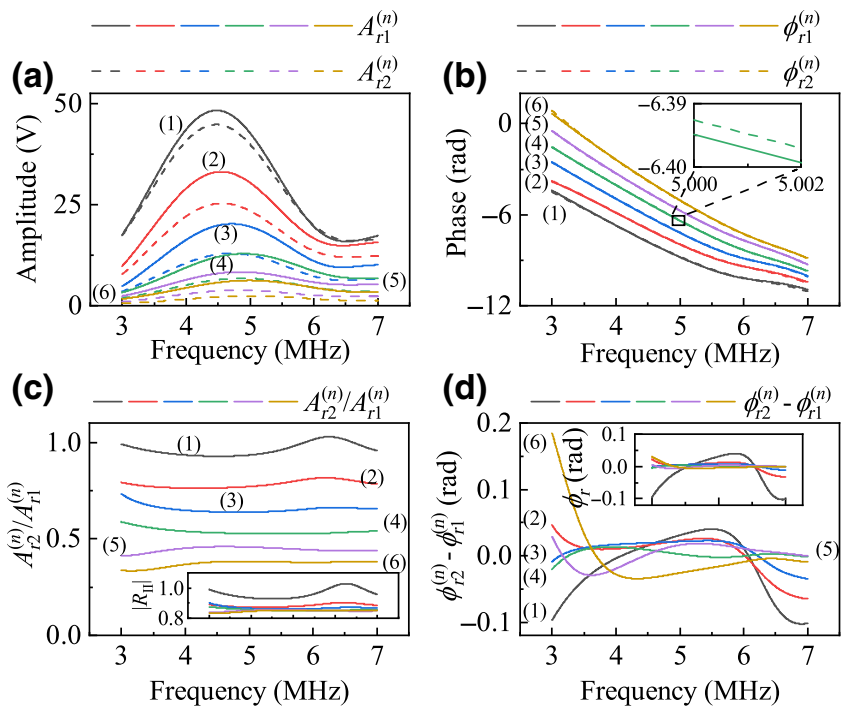

FIG. 5. The (a) amplitude and (b) phase spectra of $P_{r 1}^{(n)}(t)$ and $P_{r 2}^{(n)}(t)$ pulses for $n=1,2, \ldots, 6$; data extracted from an $L$-wave measurement on a $\left(10: 1,85^{\circ} \mathrm{C}\right)$ sample. (c),(d) Corresponding spectra of amplitude ratios (dimensionless) and phase differences.

amplitude peaks observed at approximately 4.5 MHz. Similar to the $S$-wave case, the $A_{r 1}^{(n)}(f)$ and $A_{r 2}^{(n)}(f)$ amplitudes decrease as $n$ increases. However, the difference between the amplitudes increases as $n$ increases, indicating that a higher proportion of $L$-wave energies enters PDMS than $S$ waves. In Figs. 5(c) and 5(d) we show $A_{r 2}^{(n)}(f) / A_{r 1}^{(n)}(f)$ and $\phi_{r 2}^{(n)}(f)-\phi_{r 1}^{(n)}(f)$, while $\left|R_{\mathrm{II}}(f)\right|$ and $\phi_{r}(f)$ determined from Eq. (12) are given in the insets. It should be mentioned that the $\phi_{r 2}^{(n)}-\phi_{r 1}^{(n)}$ curves in Fig. 5(d) show no systematic behavior. This can be attributed to the relatively low damping of $L$ waves in PDMS, leading to very low $\phi_{r}$ values, approximately $10^{-4}$, as shown by the $n=4,5,6$ curves in the corresponding inset. Meanwhile, the $n=$ $1,2,3$ curves deviate from expected small values due to possible pulse contaminations, which will be explained in Sec. IV A. Therefore, the phases of $P_{r 1}^{(n)}(t)$ and $P_{r 2}^{(n)}(t)$ can be neglected, and the real valued $R_{\mathrm{II}}$ leads to the $L$-wave velocity from the correspondingly simplified Eq. (2)

$$
c_{L}(f)=\frac{4 \pi \rho_{P} f}{\rho_{F} c_{F}} \frac{2\left|R_{\mathrm{II}}(f)\right|}{1-\left|R_{\mathrm{II}}(f)\right|^{2}} .
$$

\section{Processing $L$-wave data for method B}

In this method, only the $n=1$ pulses, $P_{r 2}^{(1)}(t)$ and $P_{r 3}^{(1)}(t)$, are used for computing $c_{L}(f)$ and $\alpha_{L}(f)$, whereas an absolute phase determination technique proposed by $\mathrm{He}[29,30]$ is used to obtain the phase difference between these pulses. By assuming that the largest positive peaks of $P_{r 2}^{(1)}(t)$ and $P_{r 3}^{(1)}(t)$ are located at $t_{r 2}^{(1)}$ and $t_{r 3}^{(1)}$, respectively, two 

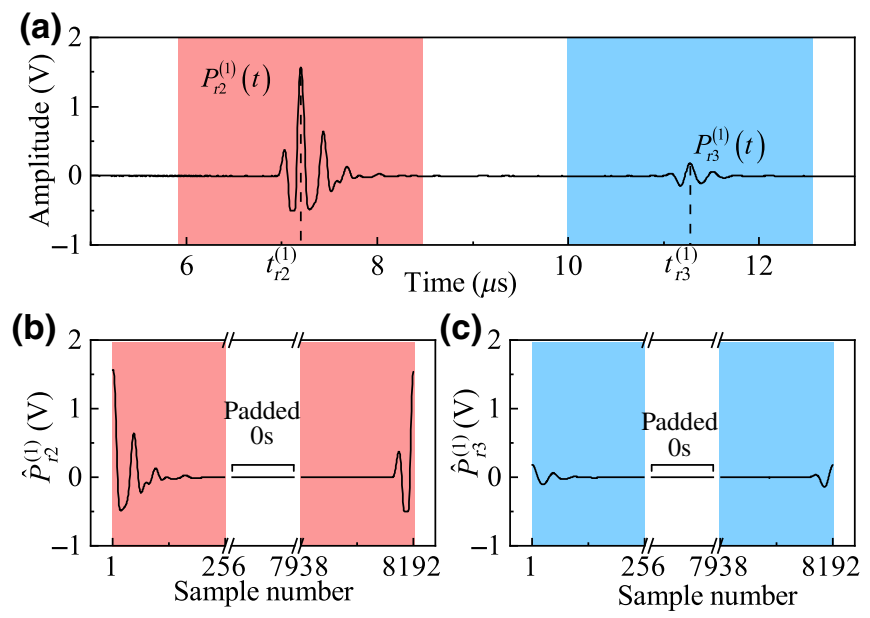

FIG. 6. Pulse shifting and zero padding the $P_{r 2}^{(n)}(t)$ and $P_{r 3}^{(n)}(t)$ pulses in (a) leads to (b) $\hat{P}_{r 2}^{(1)}(t)$ and (c) $\hat{P}_{r 3}^{(1)}(t)$.

512-point rectangular windows are center aligned with them, as highlighted in Fig. 6(a). The windowed pulses are then padded with $76800 \mathrm{~s}$ for spectrum resolution improvement, and circularly shifted to the left by 256 points. As shown in Figs. 6(b) and 6(c), the obtained signals are denoted by $\hat{P}_{r 2}^{(1)}(t)$ and $\hat{P}_{r 3}^{(1)}(t)$, and they have phase spectra $\hat{\phi}_{r 2}^{(1)}(f)$ and $\hat{\phi}_{r 3}^{(1)}(f)$. The phase difference between $P_{r 2}^{(1)}(t)$ and $P_{r 3}^{(1)}(t)$ is

$$
\phi_{r 3}^{(1)}(f)-\phi_{r 2}^{(1)}(f)=\hat{\phi}_{r 3}^{(1)}(f)-\hat{\phi}_{r 2}^{(1)}(f)-2 \pi f \Delta \tau,
$$

where $\Delta \tau=t_{r 3}^{(1)}-t_{r 2}^{(1)}$. Equation (11) is rewritten as

$$
\alpha_{P}(f)=-\frac{1}{2 D_{P}} \ln \left[\frac{-R_{\mathrm{FP}}}{T_{\mathrm{FP}} T_{\mathrm{PF}}} \frac{\hat{A}_{r 3}^{(1)}(f)}{\hat{A}_{r 2}^{(1)}(f)}\right],
$$

in which $\hat{A}_{r 2}^{(1)}(f)$ and $\hat{A}_{r 3}^{(1)}(f)$ are the amplitude spectra of $\hat{P}_{r 2}^{(1)}(t)$ and $\hat{P}_{r 3}^{(1)}(t)$, respectively.

For a single $L$-wave measurement on a $\left(10: 1,85^{\circ} \mathrm{C}\right)$ sample, the amplitude spectra of $\hat{P}_{r 2}^{(1)}(t)$ and $\hat{P}_{r 3}^{(1)}(t)$ are shown in Fig. 7(a). The values of $\hat{A}_{r 3}^{(1)}(f) / \hat{A}_{r 2}^{(1)}(f)$ are close to 0.1 , but decrease as $f$ increases (see the inset). In Fig. 7(b), the phase difference between $\hat{\phi}_{r 2}^{(1)}(f)$ and $\hat{\phi}_{r 3}^{(1)}(f)$ is readily observed. With $\hat{\phi}_{r 3}^{(1)}(f)-\hat{\phi}_{r 2}^{(1)}(f)$ shown in the upper-right inset, and the time shift between $\hat{P}_{r 3}^{(1)}(t)$ and $\hat{P}_{r 2}^{(1)}(t)$ calculated as $\Delta \tau=4.11 \mu \mathrm{s}$, the total phase difference $\phi_{r 3}^{(1)}(f)-\phi_{r 2}^{(1)}(f)$ is determined from Eq. (15) and presented in the bottom-left inset of Fig. 7(b). It is worth mentioning that the total phase difference barely depends on $\hat{\phi}_{r 3}^{(1)}(f)-\hat{\phi}_{r 2}^{(1)}(f)$ because $\phi_{r 3}^{(1)}(f)-\phi_{r 2}^{(1)}(f)$ appears nearly as a linear function of $f$. From the obtained phase difference and attenuation, $c_{L}$ is calculated from Eq. (10), and the attenuation coefficient $\alpha_{L}$ is then determined from Eqs. (8) and (16).
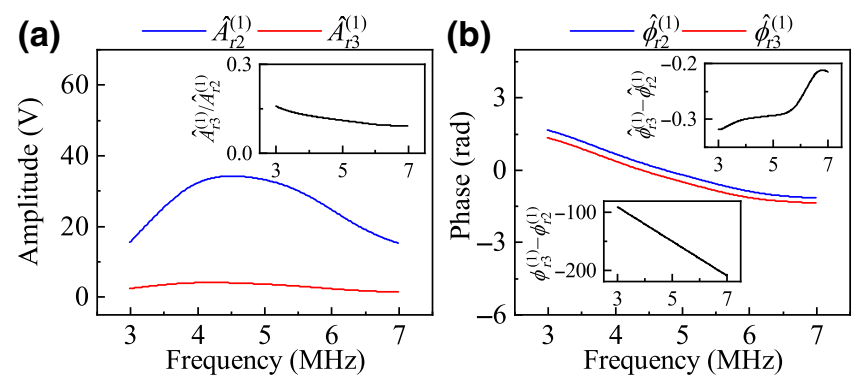

FIG. 7. The (a) amplitude and (b) phase spectra of shifted pulses $P_{r 2}^{(n)}(t)$ and $P_{r 3}^{(n)}(t)$. Data from an $L$-wave measurement on a $\left(10: 1,85^{\circ} \mathrm{C}\right)$ sample.

\section{E. The acoustic parameters of PDMS}

As explained above, although method $\mathrm{A}$ in principle is applicable to both $L$ - and $S$-wave measurements in PDMS, we use it mainly for the latter. In contrast, method B is valid only for determining the $L$-wave parameters, because the attenuation of $S$ waves induces very low SNRs in the $P_{r 3}^{(n)}(t)$ echoes.

For the five sample groups, the $S$-wave velocities as functions of $f$ obtained using method $\mathrm{A}$ are plotted in Fig. 8(a). Acoustic dispersion is observed, as $c_{S}$ increases when $f$ changes from 3.0 to $7.0 \mathrm{MHz}$. Also, $c_{S}$ is dependent on both $T$ and $\sigma$. When cured at $85^{\circ} \mathrm{C}$, samples containing more curing agents exhibit lower $c_{S}$. Among the five groups, the $\left(10: 1,100^{\circ} \mathrm{C}\right)$ group exhibits the highest $c_{S}$. The corresponding attenuation coefficient is shown in Fig. 8(b), and it has similar dispersion characteristics. However, there is no significant difference in $\alpha_{S}$ among
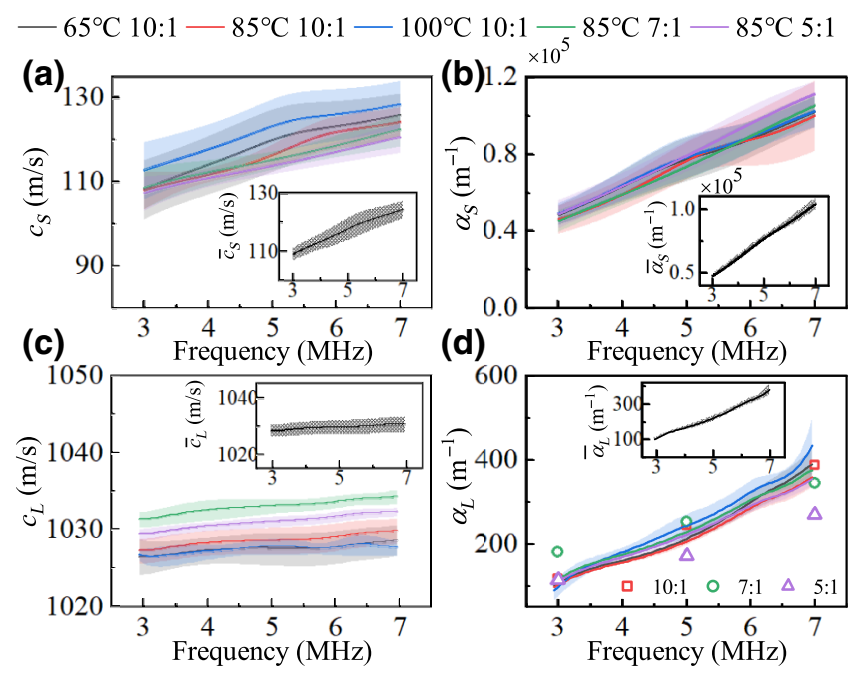

FIG. 8. Measured wave velocities and attenuation coefficients in the five sample groups. (a),(b) The coefficients $c_{S}$ and $\alpha_{S}$ measured through method A. (c),(d) The coefficients $c_{L}$ and $\alpha_{L}$ measured through method B. The scattered markers in (d) are the results reported by Tsou et al. [22], and the insets in each figure represent corresponding averaged data over all five groups. 
the different groups, i.e., $T$ and $\sigma$ barely influence $S$-wave attenuations in PDMS.

The $L$-wave velocities and attenuation coefficients measured using method B are shown in Figs. 8(c) and 8(d), respectively. In Fig. 8(c), a slight dispersion of $c_{L}$ is found within the frequency range 3.0-7.0 MHz. Using the (7:1, $85^{\circ} \mathrm{C}$ ) group as an example, $c_{L}$ increases by approximately $0.29 \%$ from $1031.3 \pm 1.0 \mathrm{~m} / \mathrm{s}$ to $1034.3 \pm 0.9 \mathrm{~m} / \mathrm{s}$. The variation in $c_{L}$ among the groups is only $0.5 \%$ between the highest value found in the $\left(7: 1,85^{\circ} \mathrm{C}\right)$ group and the lowest value found in the $\left(10: 1,100^{\circ} \mathrm{C}\right)$ group. When the mixing ratio $\sigma$ is fixed at $10: 1$, the three curves corresponding to different curing temperatures $T$ almost overlap, indicating that the curing temperature is unimportant when considering the $L$-wave velocities.

The attenuation coefficients of $L$ waves in Fig. 8(d) show a clear frequency dependence, with $\alpha_{L}$ from the (10:1, $100^{\circ} \mathrm{C}$ ) group exhibiting the strongest dependency. For the curing temperature $85^{\circ} \mathrm{C}$, the attenuation $\alpha_{L}$ is slightly dependent on the mixing ratio with $7: 1$ leading to the highest value.

\section{F. The influence of PDMS aging}

Previous studies of PDMS have shown that the properties of PDMS might change due to the aging of the material $[35,36]$. Thus, it is necessary to check whether the measured acoustic parameters also vary over long periods. Three samples taken from the $\left(10: 1,65^{\circ} \mathrm{C}\right)$ group are measured every $12 \mathrm{~h}$ after fabrication, and this process lasted until the obtained parameters became stable. For the results shown in Figs. 9(a) and 9(b), the $S$ - and $L$-wave parameters are obtained using methods A and B, respectively. We find that the $S$-wave velocity $c_{S}$ shows very little fluctuations during the 96 -hour period. The $L$-wave velocity $c_{L}$, however, increases within the first $60 \mathrm{~h}$ and then becomes stable at $1027 \mathrm{~m} / \mathrm{s}$. The $L$-wave attenuation coefficient $\alpha_{L}$ decreases within the first $60 \mathrm{~h}$ and approaches constant values of 136.8, 222.7, and $331.3 \mathrm{~m}^{-1}$ at $f=3.5,5.0$, and $6.5 \mathrm{MHz}$, respectively. Its $S$-wave counterpart $\alpha_{S}$ reaches $0.687 \times 10^{5}, 0.930 \times 10^{5}$, and $1.119 \times 10^{5} \mathrm{~m}^{-1}$ at these frequencies.

\section{DISCUSSION}

\section{A. Comments and comparisons of the methods}

Two different methods have been developed and used to obtain the frequency-dependent phase velocities and attenuation coefficients of the $L$ and $S$ waves in PDMS. Method A, generally used for $S$ waves, involves measuring the wave reflections from a solid interface, first unloaded and then with a PDMS sample attached. This method is an extension of that proposed by Frizzell et al. [32], who used a pulse superposition technique [37] to measure the magnitude and phase of the reflection coefficients. Madsen et al.
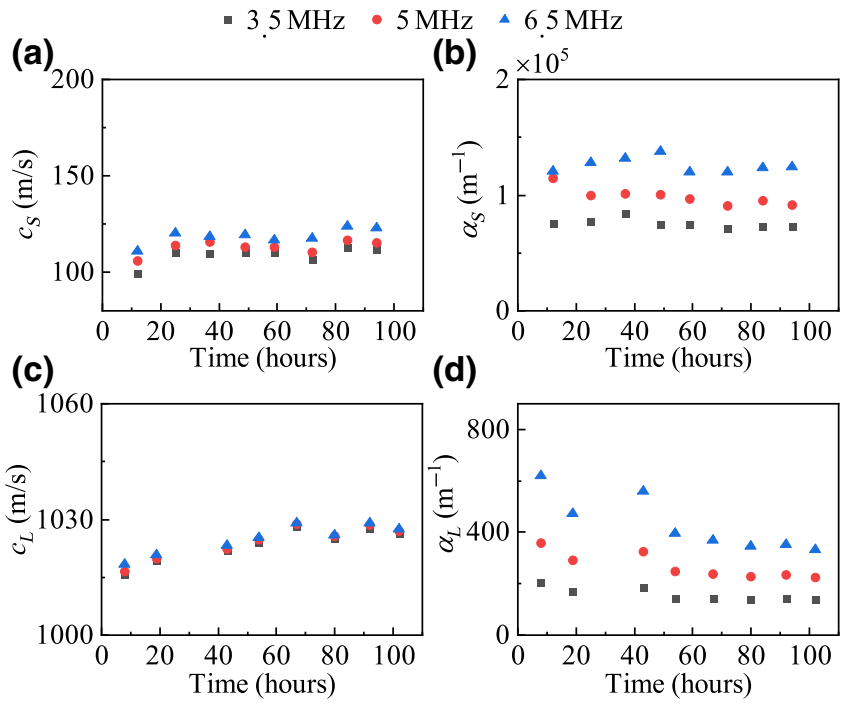

FIG. 9. The aging dependencies of the acoustic parameters along a 96-hour period: (a) $S$-wave velocity $c_{S}$, (b) $S$-wave attenuation coefficient $\alpha_{S}$, (c) $L$-wave velocity $c_{L}$, and (d) $L$-wave attenuation coefficient $\alpha_{L}$. Data averaged from nine replicate measurements on three different samples of the $\left(10: 1,65^{\circ} \mathrm{C}\right)$ group.

[25] perfected this theory and proposed a detailed experimental procedure. In this work, we introduce progresses in both theory and practice, including the use of broadband pulse signals to obtain the dispersive characteristics of the parameters in single measurements, as well as employing multiple pulses in the determination of the results through linear fittings.

Method B exploits the wave transmission characteristics in a double-layered solid-PDMS structure, and is only applicable for $L$-wave measurements. This method is much easier to carry out than the early attempts of Sachse and Pao [38], who used two transducers respectively as the source and receiver. An alternative immersion-type practice following the similar principle of Sachse and Pao was developed by He and Zheng [29,30], in which measuring the thicknesses of the samples was not necessary. However, their method required samples to be carefully placed with their surfaces exactly perpendicular to the wave paths.

The beauty of methods A and B proposed here, besides the use of only a single transducer and having an easy alignment of the configuration, lies in the introduction of an FS disc and elimination of the influence of the interfaces. On the one hand, the FS disc acts as a buffer between the transducer and the samples, which helps to avoid pulse mixing in the time domain. Specifically, the FS should be as thin as possible to ensure minimal wave attenuation, but thick enough to separate different echoes. Since $L$ waves travel faster than $S$ waves, $D_{F}$ in $L$-wave measurements is chosen to be larger than in the $S$-wave case. As shown in Fig. 2(a), the time delay between adjacent $P_{r 1}^{(n)}(t)$ echoes 


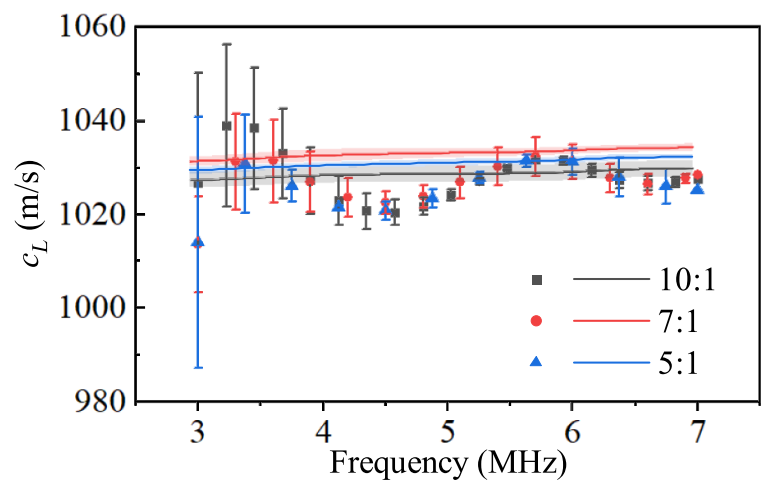

FIG. 10. Comparisons of the $L$-wave velocities obtained from methods A (data markers) and B (lines). Data of the three groups, $\left(10: 1,85^{\circ} \mathrm{C}\right),\left(7: 1,85^{\circ} \mathrm{C}\right)$, and $\left(5: 1,85^{\circ} \mathrm{C}\right)$, are averaged from nine measurements on three samples in each group.

is $\Delta \tau_{1} \approx 4.17 \mu \mathrm{s}$, while for those in Fig. 2(b) the time delay is $\Delta \tau_{2} \approx 6.60 \mu \mathrm{s}$; both helps to avoid signal contaminations on the desired wave packets. On the other hand, in both methods, the FS disc is kept in constant contact with the transducer until all measurements are complete, guaranteeing that the wave transmission characteristics at the transducer-FS interface remain unchanged. This is precisely why the properties of this interface do not appear in both theories presented in Secs. II B and II C.

According to the data analyses in Secs. III C and III D, the $L$-wave velocity $c_{L}$ can be obtained through both methods. Here, the $c_{L}$ derived from methods $\mathrm{A}$ and $\mathrm{B}$ are compared to demonstrate the consistency of the two protocols. In Fig. 10, $c_{L}(f)$ from the two methods agrees well, although the method A results show some unexpected fluctuations with respect to the frequency. The explanation for the fluctuations is that, when a PDMS sample is adhered to S1, some "harmful" pulses traveling complicated paths [such as those marked with black arrows in Fig. 2(b)] might also exist, although the thicknesses of the FS and PDMS discs are carefully selected in an effort to avoid these. As a consequence, the $P_{r 1}^{(n)}(t)$ and $P_{r 2}^{(n)}(t)$ pulses could be contaminated, which in turn cause inaccuracies in the $R_{\mathrm{II}}(f)$ calculations of method A. These fluctuations are clearer at low frequencies, because here the possible contaminants experience lower attenuations and thus play larger roles, whereas the transducer sensitivity is lower than that at the central frequency.

\section{B. Comparison with existing data}

The PDMS studied by Madsen et al. [25] was RTV 615 manufactured by the General Electric Company, which is different from the samples used here. Nevertheless, their data are the most relevant $S$-wave data that can be used for comparison. Since the curing parameters were unspecified in their work, the five curves in Figs. 8(a) and 8(b) are averaged and plotted in the insets to allow comparisons.
The averaged $\bar{c}_{S}$ within the $3.0-7.0 \mathrm{MHz}$ range increases from $109.0 \pm 2.2$ to $124.3 \pm 3.0 \mathrm{~m} / \mathrm{s}$, whereas the averaged attenuation coefficient $\bar{\alpha}_{S}$ increases from $(0.477 \pm$ $0.017) \times 10^{5}$ to $(1.042 \pm 0.044) \times 10^{5} \mathrm{~m}^{-1}$. Thus, the $c_{S}$ of Sylgard 184 is higher than that of RTV 615, the value of which was reported to be 75-95 m/s in the same frequency range [25]. The $\alpha_{S}$ values obtained by Madsen et al. were $1.1 \times 10^{5}$ to $2.4 \times 10^{5} \mathrm{~m}^{-1}$, higher than the results here but of the same magnitude.

Only a few literature studies [22-24] have reported $L$ wave parameters in PDMS, especially for different curing conditions. In Fig. 8(d), the $\alpha_{L}$ results given by Tsou et al. [22] at $f=3.0,5.0$, and $7.0 \mathrm{MHz}$ are also presented, which followed a similar frequency dependence when compared with the results obtained here. However, for samples of different mixing ratios, the differences in $\alpha_{L}$ are found to be smaller here. The dispersion of $c_{L}$ was not mentioned in their work, and only the $c_{L}$ values corresponding to different mixing ratios were presented, i.e., $1076.5,1089.1$, and $1119.1 \mathrm{~m} / \mathrm{s}$ for $10: 1,7: 1$, and 5:1 samples, respectively. Also, they did not mention the differences caused by varied curing temperatures. Their results showed that changing the mixing ratio from 10:1 to 5:1 increased $c_{L}$ by approximately $4 \%$, which is much higher than that in our case (about $0.4 \%$ ) shown in Fig. 8(c). The five curves in Figs. 8(c) and 8(d) are averaged and plotted in the insets. The averaged $L$-wave velocity $\bar{c}_{L}$ increases from $1028.3 \pm 2.1$ to $1030.6 \pm 2.7 \mathrm{~m} / \mathrm{s}$ within 3.0-7.0 MHz. The averaged value $\bar{\alpha}_{L}$ of $\alpha_{L}$ ranges from $101.0 \pm 8.2$ to $382.8 \pm 32.4 \mathrm{~m}^{-1}$.

It is interesting to note from Fig. 8(c) that, at an $85^{\circ} \mathrm{C}$ curing temperature, $c_{L}$ is highest in the $\sigma=7: 1$ group, and reduces as the mixing ratio is changed to $10: 1$ or $5: 1$. Therefore, although the recommended $\sigma$ from the manufacturer is $10: 1$, this might not be the optimized mixing ratio if a higher wave velocity is preferred. During the curing process of PDMS, complicated crosslinking reaction arises, increasing the difficulties in understanding the dependence of its acoustic properties on curing parameters. A more detailed explanation regarding this phenomena requires collaboration from both the physics and materials science communities.

\section{Acoustic dispersion of PDMS parameters}

Working frequencies of commonly used BAW and SAW devices can range from 2 to $8 \mathrm{MHz}$ and from 5 to $50 \mathrm{MHz}$ $[9,39,40]$, respectively. However, due to the availability of transducers, the measurements are only conducted within the frequency band 3.0-7.0 MHz here, which overlap with typical BAW frequencies, but only cover the lower part of the SAW bands. To provide the estimates outside the 3.0-7.0-MHz range, we present an extrapolation of data fittings based on the Kramers-Kronig dispersion relations [41]. 
TABLE III. Fitted parameters for the dispersive characteristics of the attenuation coefficients and phase velocities of $L$ and $S$ waves in PDMS.

\begin{tabular}{lcccccc}
\hline \hline & $\left(10: 1,85^{\circ} \mathrm{C}\right)$ & $\left(7: 1,85^{\circ} \mathrm{C}\right)$ & $\left(5: 1,85^{\circ} \mathrm{C}\right)$ & $\left(10: 1,65^{\circ} \mathrm{C}\right)$ & $\left(10: 1,100{ }^{\circ} \mathrm{C}\right)$ & Average \\
\hline$\alpha_{S, 5 \mathrm{MHz}}\left(\mathrm{m}^{-1}\right)$ & $7.422 \times 10^{4}$ & $7.426 \times 10^{4}$ & $7.966 \times 10^{4}$ & $7.630 \times 10^{4}$ & $7.711 \times 10^{4}$ & $7.633 \times 10^{4}$ \\
$y_{S}$ & 0.922 & 1.017 & 1.000 & 0.843 & 0.841 & 0.924 \\
$c_{S, 5 \mathrm{MHz}}\left(\mathrm{m} \mathrm{s}^{-1}\right)$ & 117.0 & 115.7 & 114.2 & 119.1 & 122.3 & 117.6 \\
$\alpha_{L, 5 \mathrm{MHz}}\left(\mathrm{m}^{-1}\right)$ & $2.148 \times 10^{2}$ & $2.347 \times 10^{2}$ & $2.255 \times 10^{2}$ & $2.238 \times 10^{2}$ & $2.449 \times 10^{2}$ & $2.288 \times 10^{2}$ \\
$y_{L}$ & 1.494 & 1.368 & 1.331 & 1.577 & 1.517 & 1.456 \\
$c_{L, 5 \mathrm{MHz}}\left(\mathrm{m} \mathrm{s}^{-1}\right)$ & $1.029 \times 10^{3}$ & $1.033 \times 10^{3}$ & $1.031 \times 10^{3}$ & $1.028 \times 10^{3}$ & $1.028 \times 10^{3}$ & $1.030 \times 10^{3}$ \\
\hline \hline
\end{tabular}

According to this principle, a power law is used to describe the dispersive behavior of the attenuation coefficients [41,42]

$$
\alpha_{i}(f)=\alpha_{i, 0}(2 \pi f)^{y_{i}} \quad \text { or } \quad \alpha_{i}(f)=\alpha_{i, 5 \mathrm{MHz}}\left(\frac{f}{5 \mathrm{MHz}}\right)^{y_{i}}
$$

where $i=L$ or $S, \alpha_{i, 0}$ is the corresponding attenuation constant, and $y_{i}$ is a positive power less than 2 [43]. Here, the reference frequency is chosen as $5 \mathrm{MHz}$, because this is the nominal central frequency of both transducers used in our experiments. The corresponding phase velocities are then given as [42]

$$
\begin{aligned}
\frac{1}{c_{i}(f)}= & \frac{1}{c_{i, 5 \mathrm{MHz}}}+\frac{\alpha_{i, 5 \mathrm{MHz}} \tan \left(y_{i} \pi / 2\right)}{2 \pi \times 5 \mathrm{MHz}} \\
& \times\left[\left(\frac{f}{5 \mathrm{MHz}}\right)^{y_{i}-1}-1\right] .
\end{aligned}
$$

After data averaging from nine replicate measurements on three different samples within each of the five groups, frequency dependencies of the attenuation coefficients $\left(\alpha_{L}\right.$ and $\left.\alpha_{S}\right)$ and phase velocities $\left(c_{L}\right.$ and $c_{S}$ ) are fitted through Eq. (17) and (18), respectively. The resultant parameters are listed in Table III.

The measured data are also respectively averaged across different sample groups because we found insignificant intergroup discrepancies with the fitted parameters given in Table III and the curves of intergroup averaged $\bar{\alpha}_{L}$ and $\bar{\alpha}_{S}$ illustrated in Figs. 11(a) and 11(b). The damping of $S$ waves follows

$$
\bar{\alpha}_{S}(f)=7.633 \times 10^{4}\left(\frac{f}{5 \mathrm{MHz}}\right)^{0.924} \mathrm{~m}^{-1}
$$

exhibiting decelerated increase as $f$ increases. This functional form is similar to the dispersion characteristics of
RTV 615 [25]. For $L$ waves, Fig. 11(b) gives

$$
\bar{\alpha}_{L}(f)=228.8\left(\frac{f}{5 \mathrm{MHz}}\right)^{1.456} \mathrm{~m}^{-1} .
$$

Therefore, the attenuation of $L$ waves in PDMS shows an accelerated increase as $f$ increases.

For the $\alpha_{L}$ values reported by Tsou et al. [22], which were scattered at several discrete frequency points, powerlaw fitting using Eq. (17) results in $y_{L}$ values of $1.42,1,12$, and 1.16 for samples of $10: 1,7: 1$, and 5:1 mixing ratios, respectively. However, the results here show that $\alpha_{L}(f)$ is nearly independent of $\sigma$.

The $c_{S}$ and $c_{L}$ values averaged over all five groups are also fitted using Eq. (18), resulting in the curves in
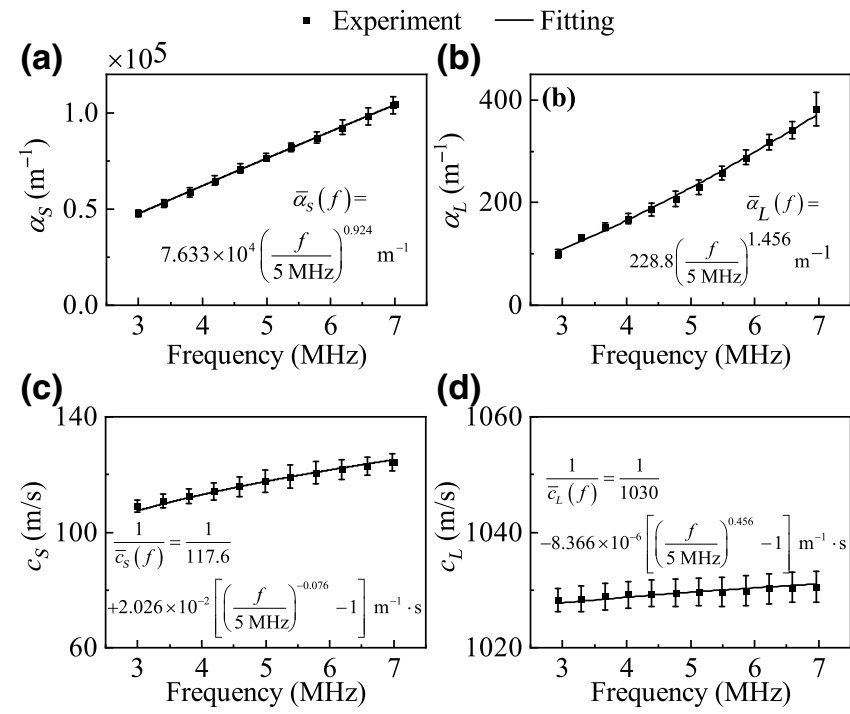

(d)

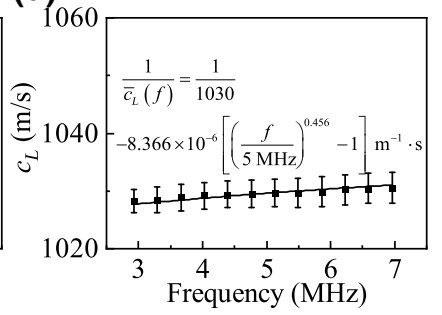

FIG. 11. Power-law fittings of the attenuation coefficients of (a) $S$ waves, and (b) $L$ waves in PDMS, and the resulting phase velocities of (c) $S$ wave and (d) $L$ waves obtained using the Kramers-Kronig principle. Source data obtained by averaging the results over the five sample groups $\left(10: 1,65^{\circ} \mathrm{C}\right),\left(10: 1,85^{\circ} \mathrm{C}\right)$, $\left(10: 1,100^{\circ} \mathrm{C}\right),\left(7: 1,85^{\circ} \mathrm{C}\right)$, and $\left(5: 1,85^{\circ} \mathrm{C}\right)$. 
TABLE IV. Complex elastic coefficients of PDMS at typical frequencies.

\begin{tabular}{lcccccc}
\hline \hline & & \multicolumn{5}{c}{$f(\mathrm{MHz})$} \\
\cline { 3 - 7 } & & 3.0 & 4.0 & 5.0 & 6.0 & 7.0 \\
\hline$C_{11}(\mathrm{GPa})$ & $\left(10: 1,85^{\circ} \mathrm{C}\right)$ & $1.088-j 0.0119$ & $1.090-j 0.0139$ & $1.091-j 0.0146$ & $1.091-j 0.0167$ & $1.093-j 0.0185$ \\
& $\left(7: 1,85^{\circ} \mathrm{C}\right)$ & $1.096-j 0.0136$ & $1.099-j 0.0158$ & $1.100-j 0.0164$ & $1.101-j 0.0181$ & $1.103-j 0.0196$ \\
& $\left(5: 1,85^{\circ} \mathrm{C}\right)$ & $1.092-j 0.0130$ & $1.095-j 0.0151$ & $1.096-j 0.0159$ & $1.097-j 0.0173$ & $1.099-j 0.0184$ \\
$C_{44}(\mathrm{MPa})$ & $\left(10: 1,85^{\circ} \mathrm{C}\right)$ & $9.777-j 5.57$ & $10.482-j 5.924$ & $10.986-j 6.789$ & $12.055-j 7.423$ & $12.546-j 7.705$ \\
& $\left(7: 1,85^{\circ} \mathrm{C}\right)$ & $9.916-j 5.57$ & $10.560-j 5.994$ & $11.021-j 6.405$ & $11.463-j 7.000$ & $11.982-j 7.677$ \\
& $\left(5: 1,85^{\circ} \mathrm{C}\right)$ & $9.437-j 5.70$ & $10.040-j 6.104$ & $10.459-j 6.533$ & $10.866-j 7.097$ & $11.378-j 7.658$ \\
\hline \hline
\end{tabular}

Figs. 11(c) and 11(d), respectively. The expressions

$$
\begin{aligned}
\frac{1}{\bar{c}_{S}(f)}= & \frac{1}{117.6}+2.026 \times 10^{-2} \\
& \times\left[\left(\frac{f}{5 \mathrm{MHz}}\right)^{-0.076}-1\right] \mathrm{m}^{-1} \mathrm{~s}
\end{aligned}
$$

and

$$
\begin{aligned}
\frac{1}{\bar{c}_{L}(f)}= & \frac{1}{1030}-8.366 \times 10^{-6} \\
& \times\left[\left(\frac{f}{5 \mathrm{MHz}}\right)^{0.456}-1\right] \mathrm{m}^{-1} \mathrm{~s}
\end{aligned}
$$

should be helpful for users who are uncertain about the sample preparation processes.

By using the power-law extrapolation based on the Kramers-Kronig principle, the main errors might come from possible inaccuracies of the obtained power index. However, using the Kramers-Kronig dispersion relationship is perhaps the only way to extend the results here to other frequencies. Also, although the above extrapolating protocol has achieved success in the characterization of biological tissues, the extrapolation of the fitted power law beyond the $3.0-7.0-\mathrm{MHz}$ band is strictly empirical and may be regarded as the estimates available at higher frequencies given state-of-the-art measurements and assuming that the attenuation mechanism remains the same.

\section{The complex elastic coefficients of PDMS}

The mechanical properties of PDMS sonicated by ultrasound is conveniently described theoretically by the constitutive relationship between stress and strain using the Voigt matrix of complex-valued elastic coefficients [17], which can be computed from the wave velocity and attenuation coefficients obtained above.

Because PDMS is an isotropic material [44], the elastic Voigt matrix contains only two independent elastic coefficients [17],

$$
C_{11}=\rho_{P} \hat{c}_{L}^{2}, \quad C_{44}=\rho_{P} \hat{c}_{S}^{2},
$$

where $\hat{c}_{L}$ and $\hat{c}_{S}$ are the complex-valued velocities of $L$ and $S$ waves that are derived from the complex wave numbers as

$$
\hat{c}_{i}=\frac{2 \pi f}{2 \pi f / c_{i}+j \alpha_{i}} \quad \text { for } i=L \text { or } S .
$$

For the sample groups $\left(10: 1,85^{\circ} \mathrm{C}\right),\left(7: 1,85^{\circ} \mathrm{C}\right)$, and $(5: 1$, $85^{\circ} \mathrm{C}$ ), the coefficients $C_{11}$ and $C_{44}$ for $f=3,4,5,6$, and 7 $\mathrm{MHz}$ are directly determined from the measured data, and the resulting values are listed in Table IV.

The attenuation coefficients and wave velocities are both frequency dependent, as are $C_{11}$ and $C_{44}$. The real parts of $C_{11}$ and $C_{44}$ increase $0.5 \%$ and $20 \%-30 \%$, respectively, as the frequency increases from $3.0 \mathrm{MHz}$ to $7.0 \mathrm{MHz}$.

The values $C_{11}=(1.035-j 0.0026) \mathrm{GPa}$ and $C_{44}=$ (4.31-j0.68) $\mathrm{MPa}$ of PDMS reported by Moiseyenko and Bruus [18] were derived from Folds [23] and Madsen [25], respectively. Their $L$-wave parameter $C_{11}=$ $(1.035-j 0.0026) \mathrm{GPa}$ is close to our results listed in Table IV, while their $S$-wave parameter $C_{44}$ is more than two times smaller than our results. This discrepancy will influence the quantitative prediction, although not the qualitative prediction of the acoustofluidic response in PDMS devices.

For users who are not working at the frequency points in Table IV, accurate $C_{11}$ and $C_{44}$ values can be obtained through Eqs. (23) and (24), where the required $c_{i}$ and $\alpha_{i}$ values should be determined from Eqs. (17) and (18) using the parameters in Table III.

\section{CONCLUSIONS}

This work reports a systematic study of the acoustic parameters of Sylgard TM 184 PDMS, where the phase velocities and the attenuation coefficients of the longitudinal and shear waves, together with the sample density, are obtained from the same groups of samples. For the five sample groups studied here, corresponding to five different sample preparation protocols, intergroup deviations are found to be insignificant, especially regarding the 
damping of both longitudinal and shear waves. The longitudinal wave velocity obtained here $(1028.3-1030.6 \mathrm{~m} / \mathrm{s})$ is slightly lower than that reported by Tsou et al. [22] (approximately 1076.5-1119.1 m/s) and Folds [23] for RTV $615(1083 \mathrm{~m} / \mathrm{s})$. Meanwhile the shear wave velocity of Sylgard ${ }^{\mathrm{TM}} 184$ in the current measurements $(109.0-124.3 \mathrm{~m} / \mathrm{s})$ is higher than that of RTV 615 reported by Madsen et al. [25] (75-95 m/s). The measured damping of longitudinal waves in Sylgard TM 184 agree well with that reported by Tsou et al. [22], but the shear wave attenuation coefficient is lower than that in RTV 615 [25]. Also, the longitudinal and shear waves obtained in this study exhibit accelerated and decelerated increasing as the frequency increases, respectively, which is well expected. Based on the classical principles of acoustics, the dispersive characteristics of the four wave parameters are extrapolated outside the $3.0-7.0-\mathrm{MHz}$ band to provide the estimates available at higher frequencies given the current state-of-the-art experimental data and assuming that the attenuation mechanism remains the same. Furthermore, the obtained elastic constants $C_{11}$ and $C_{44}$ are useful for the design and evaluations of PDMS-based acoustofluidic devices.

\section{ACKNOWLEDGMENTS}

This work was supported by the National Natural Science Foundation of China (Grants No. 11934009, No. 11974179, No. 81627802, No. 11774166, No. 11774168 , No. 11874216, and No. 11674173), Jiangsu 333 High-level Talents Training Project, and the Fundamental Research Funds for the Central Universities. X.G. acknowledges support as a Fellow at the Collaborative Innovation Center for Cardiovascular Disease Translational Medicine, Nanjing Medical University.

[1] T. M. Squires and S. R. Quake, Microfluidics: Fluid at the nanoliter scale, Rev. Mod. Phys. 77, 977 (2005).

[2] G. M. Whitesides, The origins and the future of microfluidics, Nature 442, 368 (2006).

[3] E. K. Sackmann, A. L. Fulton, and D. J. Beebe, The present and future role of microfluidics in biomedical research, Nature 507, 181 (2014).

[4] E. Sollier, C. Murray, P. Maoddi, and D. Di Carlo, Rapid prototyping polymers for microfluidic devices and high pressure injections, Lab Chip 11, 3752 (2011).

[5] J. M. Ng, I. Gitlin, A. D. Stroock, and G. M. Whitesides, Components for integrated poly (dimethylsiloxane) microfluidic systems, Electrophoresis 23, 3461 (2002).

[6] S. Noimark, R. J. Colchester, R. K. Poduval, E. Maneas, E. J. Alles, T. Zhao, E. Z. Zhang, M. Ashworth, E. Tsolaki, A. H. Chester, et al., Polydimethylsiloxane composites for optical ultrasound generation and multimodality imaging, Adv. Funct. Mater. 28, 1704919 (2018).

[7] B. Kang, J. Shin, H.-J. Park, C. Rhyou, D. Kang, S.-J. Lee, Y.-s. Yoon, S.-W. Cho, and H. Lee, High-resolution acoustophoretic $3 \mathrm{~d}$ cell patterning to construct functional collateral cylindroids for ischemia therapy, Nat. Commun. 9, 5402 (2018).

[8] J. Friend and L. Y. Yeo, Microscale acoustofluidics: Microfluidics driven via acoustics and ultrasonics, Rev. Mod. Phys. 83, 647 (2011).

[9] A. Lenshof, C. Magnusson, and T. Laurell, Acoustofluidics 8: Applications of acoustophoresis in continuous flow microsystems, Lab Chip 12, 1210 (2012).

[10] A. Ozcelik, J. Rufo, F. Guo, Y. Gu, P. Li, J. Lata, and T. J. Huang, Acoustic tweezers for the life sciences, Nat. Methods 15, 1021 (2018).

[11] W. Connacher, N. Zhang, A. Huang, J. Mei, S. Zhang, T. Gopesh, and J. Friend, Micro/nano acoustofluidics: Materials, phenomena, design, devices, and applications, Lab Chip 18, 1952 (2018).

[12] M. Wiklund, Acoustofluidics 12: Biocompatibility and cell viability in microfluidic acoustic resonators, Lab Chip 12, 2018 (2012).

[13] P. Li, Z. Mao, Z. Peng, L. Zhou, Y. Chen, P.-H. Huang, C. I. Truica, J. J. Drabick, W. S. El-Deiry, and M. Dao, et al., Acoustic separation of circulating tumor cells, Proc. Natl. Acad. Sci. USA 112, 4970 (2015).

[14] T. Laurell, F. Petersson, and A. Nilsson, Chip integrated strategies for acoustic separation and manipulation of cells and particles, Chem. Soc. Rev. 36, 492 (2007).

[15] Z. Tian, S. Yang, P.-H. Huang, Z. Wang, P. Zhang, Y. Gu, H. Bachman, C. Chen, M. Wu, Y. Xie, et al., Wave number-spiral acoustic tweezers for dynamic and reconfigurable manipulation of particles and cells, Sci. Adv. 5, eaau6062 (2019).

[16] X. Ding, P. Li, S.-C. S. Lin, Z. S. Stratton, N. Nama, F. Guo, D. Slotcavage, X. Mao, J. Shi, F. Costanzo, et al., Surface acoustic wave microfluidics, Lab Chip 13, 3626 (2013).

[17] N. R. Skov, P. Sehgal, B. J. Kirby, and H. Bruus, ThreeDimensional Numerical Modeling of Surface-AcousticWave Devices: Acoustophoresis of Micro- and Nanoparticles Including Streaming, Phys. Rev. Appl. 12, 044028 (2019).

[18] R. P. Moiseyenko and H. Bruus, Whole-System Ultrasound Resonances as the Basis for Acoustophoresis in All-Polymer Microfluidic Devices, Phys. Rev. Appl. 11, 014014 (2019).

[19] N. R. Skov, J. S. Bach, B. G. Winckelmann, and H. Bruus, 3D modeling of acoustofluidics in a liquid-filled cavity including streaming, viscous boundary layers, surrounding solids, and a piezoelectric transducer, AIMS Math. 4, 99 (2019).

[20] Z. Ni, C. Yin, G. Xu, L. Xie, J. Huang, S. Liu, J. Tu, X. Guo, and D. Zhang, Modelling of saw-pdms acoustofluidics: Physical fields and particle motions influenced by different descriptions of the pdms domain, Lab Chip 19, 2728 (2019).

[21] N. Skov and H. Bruus, Modeling of microdevices for saw-based acoustophoresis study of boundary conditions, Micromachines 7, 182 (2016).

[22] J. K. Tsou, J. Liu, A. I. Barakat, and M. F. Insana, Role of ultrasonic shear rate estimation errors in assessing inflammatory response and vascular risk, Ultrasound Med. Biol. 34, 963 (2008). 
[23] D. Folds, Speed of sound and transmission loss in silicone rubbers at ultrasonic frequencies, J. Acoust. Soc. Am. 56, 1295 (1974).

[24] K. Zell, J. Sperl, M. Vogel, R. Niessner, and C. Haisch, Acoustical properties of selected tissue phantom materials for ultrasound imaging, Phys. Med. Biol. 52, N475 (2007).

[25] E. L. Madsen, H. J. Sathoff, and J. A. Zagzebski, Ultrasonic shear wave properties of soft tissues and tissuelike materials, J. Acoust. Soc. Am. 74, 1346 (1983).

[26] L. Johansson, J. Enlund, S. Johansson, I. Katardjiev, and V. Yantchev, Surface acoustic wave induced particle manipulation in a pdms channel-principle concepts for continuous flow applications, Biomed. Microdevices 14, 279 (2012).

[27] T. Pritz, Frequency dependences of complex moduli and complex poisson's ratio of real solid materials, J. Sound Vibr. 214, 83 (1998).

[28] J. L. Rose, Ultrasonic Waves in Solid Media (Cambridge University Press, Cambridge, 2000).

[29] P. He, Measurement of acoustic dispersion using both transmitted and reflected pulses, J. Acoust. Soc. Am. 107, 801 (2000).

[30] P. He and J. Zheng, Acoustic dispersion and attenuation measurement using both transmitted and reflected pulses, Ultrasonics 39, 27 (2001).

[31] N. Nama, R. Barnkob, Z. Mao, C. J. Kähler, F. Costanzo, and T. J. Huang, Numerical study of acoustophoretic motion of particles in a pdms microchannel driven by surface acoustic waves, Lab Chip 15, 2700 (2015).

[32] L. A. Frizzell, E. L. Carstensen, and J. F. Dyro, Shear properties of mammalian tissues at low megahertz frequencies, J. Acoust. Soc. Am. 60, 1409 (1976).

[33] R. Dixon, Photoelastic properties of selected materials and their relevance for applications to acoustic light modulators and scanners, J. Appl. Phys. 38, 5149 (1967).
[34] A. Darinskii, M. Weihnacht, and H. Schmidt, Computation of the pressure field generated by surface acoustic waves in microchannels, Lab Chip 16, 2701 (2016).

[35] D. T. Eddington, W. C. Crone, and D. J. Beebe, in Proceedings of the 7th International Conference on Miniaturized Chemical and Biochemical Analysis Systems (Squaw Valley, California, USA, 2003), p. 1089.

[36] V. Placet and P. Delobelle, Mechanical properties of bulk polydimethylsiloxane for microfluidics over a large range of frequencies and aging times, J. Micromech. Microeng. 25, 035009 (2015).

[37] H. McSkimin, Pulse superposition method for measuring ultrasonic wave velocities in solids, J. Acoust. Soc. Am. 33, 12 (1961).

[38] W. Sachse and Y.-H. Pao, On the determination of phase and group velocities of dispersive waves in solids, J. Appl. Phys. 49, 4320 (1978).

[39] I. Leibacher, P. Reichert, and J. Dual, Microfluidic droplet handling by bulk acoustic wave (baw) acoustophoresis, Lab Chip 15, 2896 (2015).

[40] A. Salari, S. Appak-Baskoy, M. Ezzo, B. Hinz, M. C. Kolios, and S. S. H. Tsai, Dancing with the cells: Acoustic microflows generated by oscillating cells, Small 16, 1903788 (2019).

[41] M. O'Donnell, E. T. Jaynes, and J. G. Miller, KramersKronig relationship between ultrasonic attenuation and phase velocity, J. Acoust. Soc. Am. 69, 696 (1981).

[42] T. L. Szabo, Time domain wave equations for lossy media obeying a frequency power law, J. Acoust. Soc. Am. 96, 491 (1994).

[43] T. L. Szabo and J. Wu, A model for longitudinal and shear wave propagation in viscoelastic media, J. Acoust. Soc. Am. 107, 2437 (2000).

[44] A. Mata, A. J. Fleischman, and S. Roy, Characterization of polydimethylsiloxane (pdms) properties for biomedical micro/nanosystems, Biomed. Microdevices 7, 281 (2005). 